\title{
Multimetallic Oxynitrides Nanoparticles for a New Generation of Photocatalysts
}

\author{
Tingke Rao ${ }^{\mathrm{a}}$, Maria Luisa Saladino ${ }^{\mathrm{b}}$, Yuanxing Fang ${ }^{\mathrm{c}}$, Xinchen Wang ${ }^{\mathrm{c}}$, Cristina Giordano ${ }^{\star}, \mathrm{a}$
}

\begin{abstract}
A versatile synthetic strategy for the preparation of multimetallic oxy-nitrides has been designed and here exemplarily discussed considering the preparation of nanoscaled zinc-gallium oxy-nitrides and zinc-gallium-indium oxy-nitrides, two important photo-catalysts of new generation, proven to be active in key energy related processes from pollutant decomposition to overall water splitting. The synthesis presented here allows the preparation of small nanoparticles (less than $20 \mathrm{~nm}$ in average diameter), well-defined in size and shape, yet highly crystalline and with the highest surface area reported so far (up to $\sim 80 \mathrm{~m}^{2} / \mathrm{g}$ ). X-rays diffraction study shows that the final materials is not a mixture of the single oxides but a distinctive compound. The photocatalytic properties of the oxy-nitrides have been tested toward the decomposition of an organic dye (as a model reaction for the decomposition of air pollutants), showing better photocatalytic performances than the corresponding pure phases (reaction constant $0.22 \mathrm{~h}^{-1}$ ), while almost no reaction was observed in absence of catalyst or in the dark. Photocatalysts have been also tested for $\mathrm{H}_{2}$ evolution (semi-reaction of the water splitting process) with results comparable to the best literature values but leaving more room for further improvement.
\end{abstract}

\section{Introduction}

In a world in constant evolution, scientists have the delicate issue to provide suitable materials to fulfil the contemporary necessities. This is especially important in energy related matter, where valid alternatives to current systems must still be found. In the search for novel materials two main strategies can be pursued: improving already known materials or exploring novel possibilities. In this respect, nanosized materials represent an eclectic world, where one can draw on and where old and new materials still await to be (re)discovered. Thanks to their high surface area and tuneable properties depending on their size, nanomaterials can be considered new functional systems. Among these, the so-called metal oxy-nitrides (MON) have been receiving increasing

[a] Tingke Rao, Dr. Cristina Giordano

School of Biological and Chemical Science

Chemistry Department, Queen Mary University of London, Mile End Road, London E1 4NS, United Kingdom

E-mail: c.giordano@qmul.ac.uk

[b] Prof. Maria Luisa Saladino

Dipartimento Scienze e Tecnologie Biologiche, Chimiche e Farmaceutiche - STEBICEF and INSTM UdR - Palermo, Università di Palermo, Viale delle Scienze pad. 17, Palermo, I-90128 Italy

[c] Prof. Xinchen Wang, Prof Yuanxing Fang, State Key Laboratory of Photocatalysis on Energy and Environment, College of Chemistry, Fuzhou University, Fuzhou 350002, P. R. China

Supporting information for this article is given via a link at the end of the document. attention after the discovering that their electronic and optical properties can be adjusted by changing the band gap. Thus, oxides possess wide band gaps and many of them are insulators, while the band gaps of metal nitrides are narrower and the materials usually show metallic behaviour. The band gaps of MON have intermediate values, nicely placed between pure $\mathrm{MO}_{\mathrm{x}}$ and $\mathrm{MN}$ and tunable by $\mathrm{N}$-loading contents. Domen et al. have shown that the incorporation of $\mathrm{N}$ in TaON based systems can deeply change their optical and electronic properties, where a systematic increase of the $\mathrm{N}$ loading in the crystal structure can reduce the band gap ${ }^{[1]}$. Since then, more complex compositions have been explored, going from simple mono-metallic compounds to designed multi-metallic systems. Properties of the metal oxy-nitrides arise from the simultaneous presence in the crystal lattice of nitride and oxide ions, where the substitution of $\mathrm{O}^{2-}$ with $\mathrm{N}^{3-}$ induces the substitution of $\mathrm{M}^{2+}$ with $\mathrm{M}^{3+}$ and possible anions vacancies formation, to keep neutrality ${ }^{[2]}$.

Thanks to this peculiar structure, where the orbitals $2 p$ orbitals of $\mathrm{N}$ and/or $\mathrm{O}$ overlap the $\mathrm{s}, \mathrm{p}, \mathrm{d}$ orbitals of the metals causing the dispersion of the valence and conduction bands ${ }^{[3]}$, MON are photo-responsive and are thus considered photocatalysts of new generation, where the electronic structure, including band gap can be adjusted by tuning the ratio of the compositions.

Photo-catalysts are semiconductor materials able to be activated by exposure of suitable wavelength, usually in the UV-vis range. The formation of a hole/electron pair, upon light absorption, aids the processing of a reaction of interest. Domen et al. ${ }^{[4]}$ have nicely shown that GaN:ZnO solid solutions are active photo-catalysts for overall water splitting, a key processes that might alleviate the energy crisis by producing high potential molecules, such as $\mathrm{H}_{2}$, in a clean way. More recently, also Domen group ${ }^{[5]}$, has shown that $\mathrm{ZnGaON}$ in ZnO:ZnGaON nanowire-array-on-a-film photoanode could bring to stable and efficient sunlight water splitting without the assistance of any co-catalyst. By adding zinc and oxygen dopants, the quaternary nanoparticles possess a band gap of 2.6-2.8 eV $\mathrm{eV}^{[4]}$, which is narrower than the band gap of pure GaN nanoparticle $(3.4 \mathrm{eV})^{[4]}$ or $\mathrm{ZnO}(3.25 \mathrm{eV})$ and strongly depends on the zinc/gallium ratio (increasing by decreasing the metals' ratio) ${ }^{[4]-[6]}$

A band gap energy of $2.13 \mathrm{eV}$ was achieved for a $\mathrm{Zn}$-rich $\mathrm{Ga}$ oxynitride with $\mathrm{Zn} / \mathrm{Ga}=0.9 / 0.110$. The $\mathrm{Zn}_{\mathrm{x}} \mathrm{Ga}_{1-\mathrm{x}} \mathrm{O}_{\mathrm{x}} \mathrm{N}_{1-\mathrm{x}}$ solid solution with $x=0.20$ exhibited a band gap energy of around 2.43 $\mathrm{eV} .{ }^{[6]}$ The band gap energy was smaller than those for GaN and $\mathrm{ZnO}$, and has been interpreted as an enhanced valence-band maximum caused by a repulsion of $\mathrm{N}-2 \mathrm{p}$ and $\mathrm{Zn}-3 \mathrm{~d}$ orbitals ${ }^{[4]}$. One of the ways of shifting the photo-catalytic activity from the UV to the visible region is by narrowing the band gap, which can be obtained by doping In into the ZnGaON crystal structure. The band gaps decrease from $\mathrm{GaN}$ to $\operatorname{InN}$ with the cation atomic conductive d orbital energies decreasing. ${ }^{[7]}$ Due to the $\operatorname{InN}$ smaller band gap $(0.7 \mathrm{eV})^{[8]}$, the zinc-gallium-indium oxynitride (ZGIN) 
nanoparticle is expected to have smaller band gap and thus a greater photo-catalytic activity.

Tuning the band gap is especially important as it enables $\mathrm{Zn}-\mathrm{Ga}$ oxynitride nanoparticle to harvest the most of incident visible light in photo-catalytic reactions, while the introduction of $\mathrm{Zn}$ increases the photo-activity, probably by reducing the number of defects (i.e. the probability of electron-hole recombination) and by increasing the crystallinity of the final material ${ }^{[4]}$. Therefore, high crystallinity of the final material is a key property for an improved activity. The other challenge to face by preparing $\mathrm{Zn}$ - and In-based material is to avoid their volatilization, which also reduces photo-activity. ${ }^{[9]}$ Currently, the ZnO:GaN solid solutions are always prepared by nitridation of the mixed oxide commonly via co-precipitation and more recently via sol-gel based process, however so far always under $\mathrm{NH}_{3}$ flow as nitrification agent. [9], [10]

Used starting materials are the single or mixed oxides [11], [12], [13], [14], hydroxide ${ }^{[15]}$, gel-like precursors ${ }^{[16]}, \mathrm{Zn} / \mathrm{Ga} / \mathrm{CO}_{3}$ layered double hydroxides ${ }^{[17]}$ or ammonolysis of amorphous precursors ${ }^{[18]}$. Even when urea was used as nitrogen source, the synthesis required the preparation of hydroxides, which were treated at $900{ }^{\circ} \mathrm{C}$ under ammonia flow ${ }^{[19]}$. To enhance the $\mathrm{Zn}$ content a topotactic transformation was employed but under dangerous reaction conditions (ammonia in the presence of oxygen) ${ }^{[20]}$. In both cases, the final product was morphologically not very welldefined.

Less rich is the list of papers reporting the synthesis of ZGION nanoparticles and it includes the work of Kamata et al..$^{[9]}$ and Miyaake et al. ${ }^{[21]}$ both using nitridation of the metal oxide under ammonia flow and in both cases with nanoparticles not welldefined or poorly crystalline or too big. However, $\mathrm{NH}_{3}$ is toxic and most of the proposed synthesis are not suitable for large scale production.

In the present work we prepare multi-metallic oxynitrides in an easier way, via the so-called Urea-Glass-Route (UGR), which allows the incorporation of more than one metal into the oxynitrides structure, does not involve the prior preparation of the pure oxides or nitrides and does not require the use of ammonia. The starting material is a gel-like precursor, where the composition can be chosen as whished and, upon suitable heat treatment under $\mathrm{N}_{2}$ flow, leads to a homogeneous system of small yet well-defined (in size and shape) nanoparticles, highly crystalline and with very high surface area (up to $\sim 80 \mathrm{~m}^{2} / \mathrm{g}$ ). The lower temperature used helps to hinder $\mathrm{Zn}$ and $\mathrm{In}$ volatilization. For a better comparison, also pure $\mathrm{ZnO}$ and $\mathrm{GaN}$ have been prepared with the same procedure. This is, at the best of our knowledge, the first time that such versatility is achieved with one synthetic path.

For simplicity, the wording "ZGON" and "ZGION", will be used in the following to indicate the simultaneous presence of $Z n, G a$ or $\mathrm{Zn}, \mathrm{Ga}, \mathrm{In}$ atoms in the final oxy-nitride. The nominal ratio between the metals will be always indicated as $R_{M}$ or $R_{M}^{\prime}$ $\left(R_{M}=G a / Z n\right.$ or $\left.R_{M}^{\prime}=G a / / n\right)$.

\section{Results and Discussion}

The thermally treated samples have been studied via powder $X$ rays diffraction (XRD). In Figure 1, the patterns of the metallic binary and ternary samples are reported ( $Z G O N, R=10$ and $R_{M}=2$; $\mathrm{ZGION}, \mathrm{R}=10, \mathrm{R}_{\mathrm{M}}=2$ and $\mathrm{R}_{\mathrm{M}}{ }^{\prime}=2$ ) alongside those for pure $\mathrm{ZnO}$ and GaN (also prepared via UGR). The pattern obtained from the physical mixture (prepared grinding together the $\mathrm{ZnO}$ and $\mathrm{GaN}$ powders) can be described by the two separated crystalline phases, also reported for comparison. From this figure it can be seen that both $\mathrm{GaN}$ and $\mathrm{ZnO}$ phase prepared via UGR nicely match those expected from the database (see also figure SI.1). This result is not surprising for GaN, which has been already

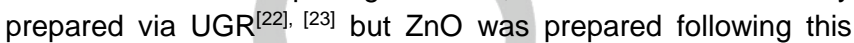
route here for the first time. The usefulness of the UGR to prepare metal oxide nanoparticles was also recently shown. ${ }^{[24]}$ The bi- and tri-metallic phase is also present as hexagonal (wurtzite type) phase with different features corresponding to the (100), (002), (101), (102), (110), (103), (112), and (201) crystallographic planes. However, it differs from the pure GaN phases both in peaks position and/or intensities. No XRD features corresponding to $\mathrm{ZnO}$ phase and mixed phase are observed, confirming the insertion of $\mathrm{Zn}$ in the $\mathrm{GaN}$ lattice.

The peaks of the multi-metallic phase are in fact observed between those of the pure phases and are very different from those of the physical mixture, where the overlapping of the $\mathrm{ZnO}$ and $\mathrm{GaN}$ peaks can be easily noted. This finding clearly indicate that the multi-metallic phases are not a physical mixture of the pure oxide and nitride but form a distinct compound.

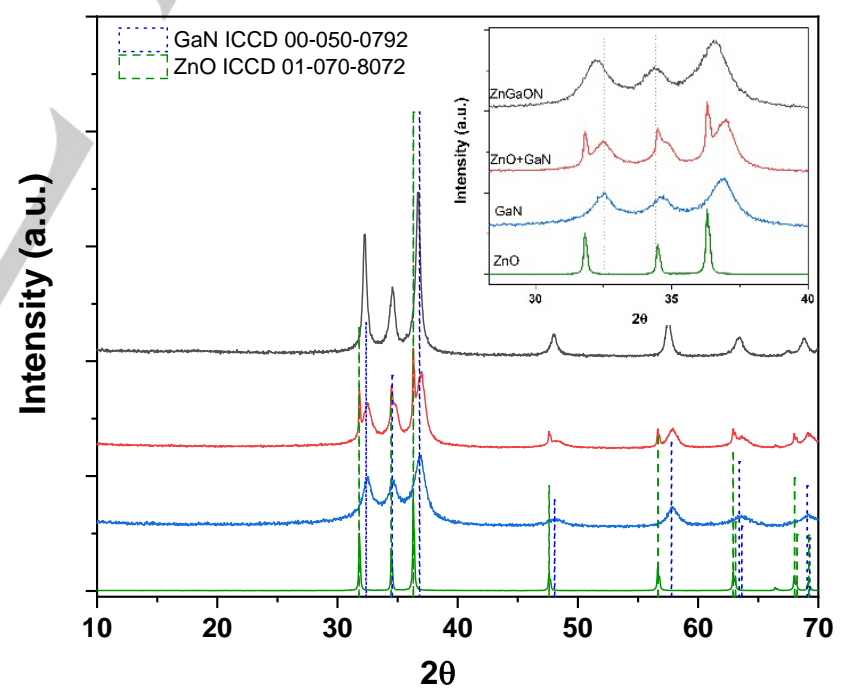

Figure 1. $X R D$ pattern of $Z G O N\left(R=10\right.$ and $\left.R_{M}=2\right)$ and $Z G I O N\left(R=10\right.$ and $R_{M}=2$ $\left.R_{M}{ }^{\prime}=2\right)$ compared with the pattern of pure $\mathrm{GaN}$ and $\mathrm{ZnO}$ powder, also prepared via UGR at $750^{\circ} \mathrm{C}$. The pattern of the physical mixture powder $(\mathrm{ZnO}+\mathrm{GaN})$ is also shown for comparison. The black vertical dotted lines in the inset are just guidelines for eyes to show the peak shift.

We have observed that for lower $R_{M}$ (namely $R_{M}=1$, i.e. equimolar ratio between the metals) peaks position is similar to the one of pure GaN (figure SI.2), while a higher $\mathrm{R}_{\mathrm{M}}$ (i.e. higher amount of gallium) shifts to the peaks at lower angles (compare to those of 
pure $\mathrm{GaN}$ ). This was previously observed and attributed to the inclusion in the lattice of bigger $\mathrm{Zn}^{2+}$ ions (compared to the smaller $\mathrm{Ga}^{3+}$ ions) that leads to its expansion. ${ }^{[25]}$ On the other hand, the further incorporation of indium ions seems to have a minor effect on the position of peaks, possibly due to the little In loading). XRD patterns of ZGION samples prepared with different In loading are reported in the SI (figure SI.3). In fact, ZGION peaks are observed at lower angles compared to pure GaN as expected (expanded structure due to incorporation of bigger ions, but higher angles compared to ZGON, which is somehow surprising considering that $\mathrm{In}^{+3}$ is bigger than $\mathrm{Ga}^{+3}$ and $\mathrm{Zn}^{+2}(80,62$ and $74 \mathrm{pm}$, respectively).

XRD patterns have been analysed by means of Rietveld method. ${ }^{[26], ~[27] ~ T h e ~ r e s u l t s ~ o f ~ c e l l ~ p a r a m e t e r ~ f o r ~ t h e ~ G a N ~ p h a s e ~}$ and crystallite size and lattice microstrain are reported in Table SI.1. The trend of cell parameters cannot be taken into account considering the crystallographic structure of GaN (wurtzitic) ${ }^{[28], ~[29], ~}$ and interesting the cell parameter a seems to be affected more by $\mathrm{R}_{\mathrm{M}}$ than $\mathrm{R}$.

It worth to note the fact that no secondary phases or residual products are observed, including the pure mono-metallic phases or the normally observed spinel phases $\left(\mathrm{ZnGa}_{2} \mathrm{O}_{4}\right)$. In order to have information on the reaction mechanism, samples were quenched at lower temperature and the corresponding $\mathrm{XRD}$ patterns are reported in Figure 2 (for $\mathrm{R}_{\mathrm{M}}=2$ ) and figure $\mathrm{SI} .4$ (for $R_{M}=1$ ). These study clearly indicated that for $T<650^{\circ} \mathrm{C}$ (regardless of $R_{M}$ ), the structure is closer to that of pure $\mathrm{ZnO}$ (although peaks of a secondary phase are also observed), somehow suggesting that the formation of the oxide phase takes place first and then the incorporation of $\mathrm{Ga}$ ions follows in a second step and only at temperature above $700{ }^{\circ} \mathrm{C}$. Similar result has been observed by Armetta et $a l .{ }^{[30]}$ for the formation of yttrium aluminum garnet (YAG) nanoparticles prepared by co-precipitation in microemulsion and calcining at high temperature by the Kirkendall effect. $^{[31]}$ The formation of an oxide intermediate phase using UGR has been also previously observed for the synthesis of TiN. ${ }^{[22]}$

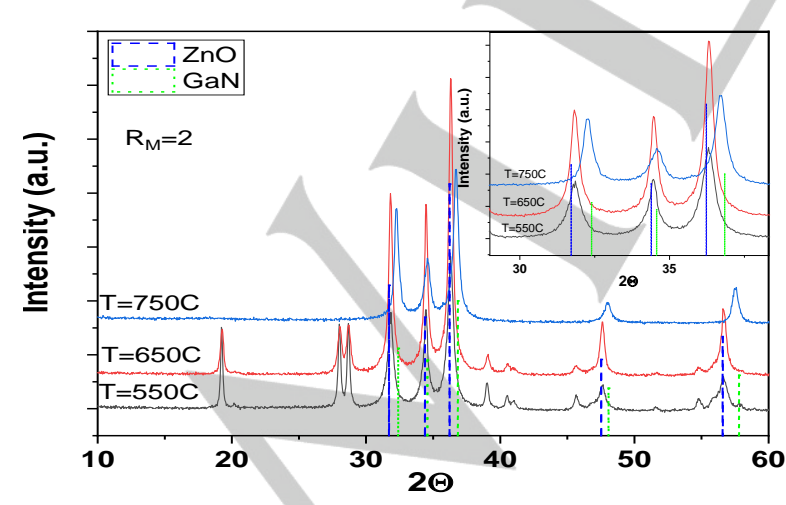

Figure 2. $X R D$ pattern of $Z G O N$ synthesized from $R=10$ and $R_{M}=2$ and treated at different temperature $\left(550,650\right.$ and $\left.750^{\circ} \mathrm{C}\right)$. The pattern of $\mathrm{ZnO}$ and $\mathrm{GaN}$ from the database are also shown for comparison as vertical lines.
The influence of urea/metal molar ratio $(\mathrm{R})$ was also studied; this ratio was in fact found to be decisive to control not only composition but also crystallinity and morphology. ${ }^{[32],[33]}$ The XRD patterns of the bimetallic phase prepared with three different urea/metal ratio and $\mathrm{R}_{\mathrm{M}}=2$ (figure $\mathrm{SI} .5$ ) shows that for lower ratios $(R \leq 5)$ the typical wurtzite-like pattern is formed but alongside a secondary phase. For $\mathrm{R} \geq 10$ the wurtzite-like phase becomes more defined and the secondary phase is no longer observed. Further increase in $\mathrm{R}(\mathrm{R} \geq 15)$ leads to sharper peaks (more crystalline structure). It must be noted that the secondary phase observed for $\mathrm{R}=5$ is not the same phase observed in samples quenched at lower temperature (see figure SI.6), which was attributed to $\mathrm{ZnGa}_{2} \mathrm{O}_{4}$ (ICDD 96-400-1768).

The effect of larger amounts of urea (higher R) seems to have little impact on the final crystallite size (assumed to be spherical and estimated by the Scherrer equation, see table 1) but precursor's composition has a decisive effect on the morphology of the metallic binary phase and homogeneity. Figure 3 shows SEM micrographs of samples prepared at different urea/metals ratio. In each case, homogeneous system can be observed, however, increasing $\mathrm{R}$, seems to bring more defined and just slightly bigger particles, which would explain the smaller surface area found for the $\mathrm{R}=15$ sample.
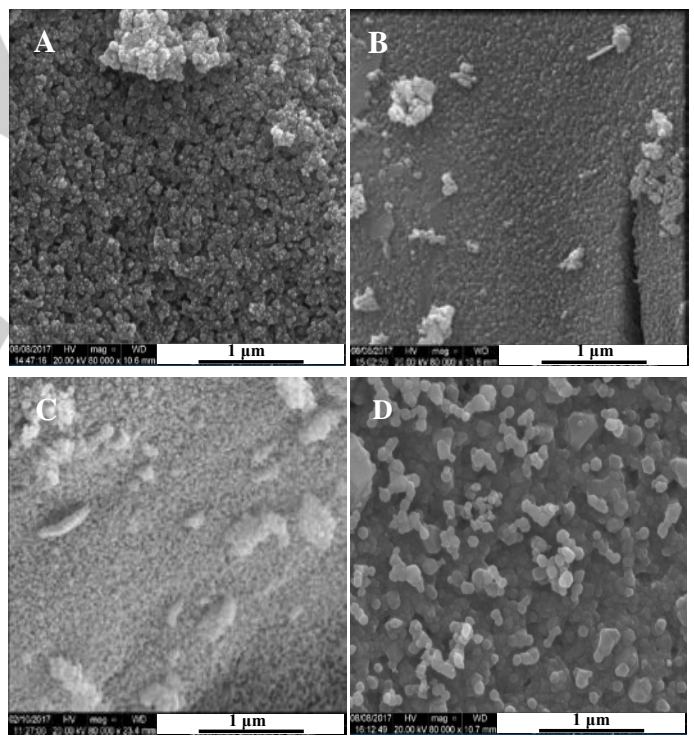

Figure 3. SEM micrographs of metallic binary phase synthesized at $R_{M}=2$ and urea ratio of: A) 8, B) 10, C) 12 and D) 15

TEM analysis (figure 4 A-B) of the as prepared bimetallic phase shown quasi-spherical particles but the sample was mainly illdefined in size and shape, and particles highly aggregated. To overcome this drawback and also to increase the surface area of the final material (i.e. maximizing its surface properties for use as photo-catalyst), the use of additives was considered and, in particular, the addition of small amount of $\mathrm{NH}_{4} \mathrm{Cl}$ was found to be beneficial. Samples prepared with the addition of $\mathrm{NH}_{4} \mathrm{Cl}$ shown a homogeneous texture, a lower degree of aggregation and a more defined morphology (figure $4 \mathrm{C}-\mathrm{D}$ ). In this figure TEM and SEM 
images of the sample prepared with and without the assistance of additives are reported.
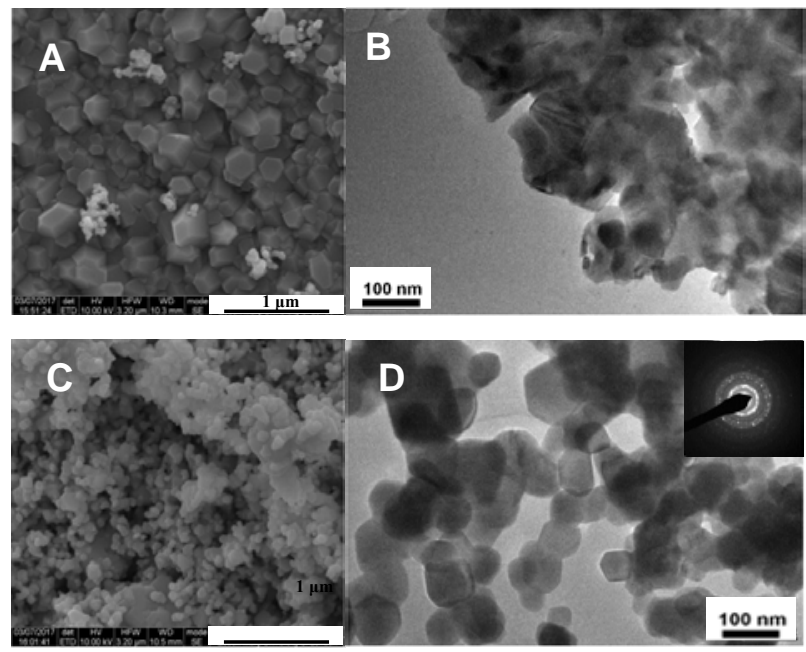

Figure 4. SEM $(A, C)$ and TEM $(B, D)$ micrographs of $Z G O N\left(R_{M}=2, R=10\right)$ prepared at $750^{\circ} \mathrm{C}$ without $(A, B)$ and with $(C, D)$ the assistance of $\mathrm{NH}_{4} \mathrm{Cl}$.

For this reason, unless otherwise specified, the samples presented in the following were all prepared with the addition of $\mathrm{NH}_{4} \mathrm{Cl}$. To ascertain the simultaneous presence of both metals in the same cluster, elemental mapping has been undertaken and results reported in figure 5 . The presence of $\mathrm{Ga}, \mathrm{Zn}, \mathrm{N}$ and $\mathrm{O}$ in the original cluster (black and white image) can be seen from this figure.

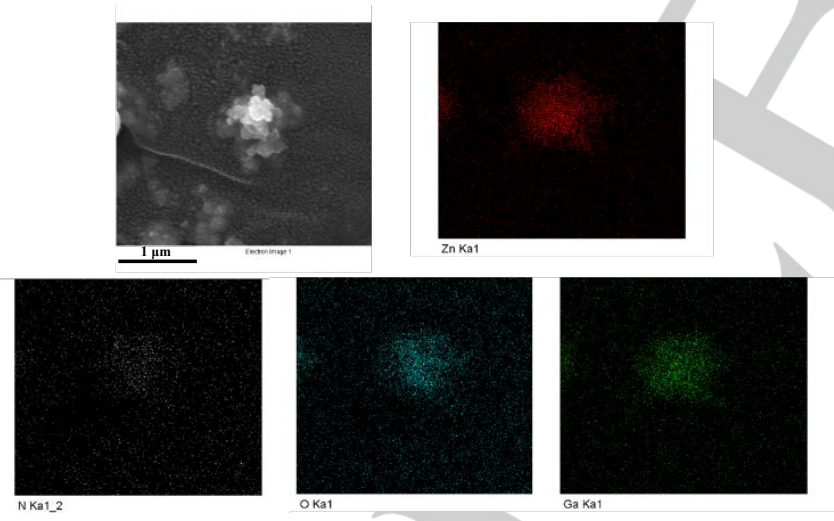

Figure 5. Elemental Mapping of $Z G O N$ with $R_{M}=2$ and Urea:Zn ratio $R=10$.

In order to have further information on the formation mechanism, $\mathrm{IR}$ investigation on the precursors at different $\mathrm{R}$ has been conducted. From the IR peaks attribution, peaks at $2200 \mathrm{~cm}^{-1}$ belongs to the $-\mathrm{CH}_{3}$ groups, from residual solvent (ethanol), in fact all samples have been dried in a vacuum oven overnight before recording IR spectra. We assume that some ethanol molecules were trapped in the metal-urea complex network as previously observed. ${ }^{[22]}$
In figure $6 \mathrm{~A}$ the IR spectrum of $\mathrm{Zn}: \mathrm{Ga}:$ Urea precursor at different Urea/Metals ratio $(R)$ and $R_{M}=2$ is reported. In figure 6.B, the IR spectrum of single metal:urea precursors are reported together with the spectra of pure urea and the bimetallic phase, for comparison.

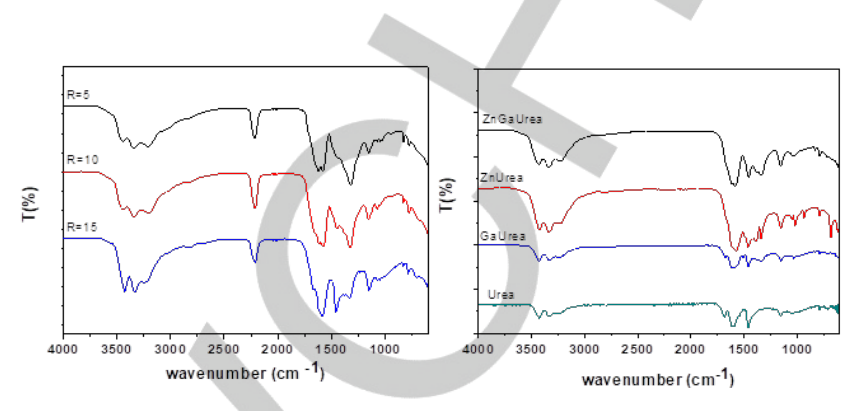

Figure 6. A) IR spectra of Zn:Ga:Urea precursor at different Urea/Metals ratio (R) and $R_{M}=2$; $B$ ) IR spectra of single metal:urea precursors and spectra of pure urea and the bimetallic phase reported for comparison.

A complete peak attribution is reported in Table SI. 2. Peaks near 1455,1630 and $1670 \mathrm{~cm}^{-1}$ corresponding to $\mathrm{C}-\mathrm{N}, \mathrm{N}-\mathrm{H}$ and $\mathrm{C}=\mathrm{O}$ bonds are present. These gradually enhanced peaks meet with the increasing of the urea ratio from 5, 10 and 15. For peaks near 3360,3230 and $3360 \mathrm{~cm}^{-1}$, these are bonds corresponding to a nitrate component in the urea nitrate sol-gel precursor.

\section{Band gap calculation}

The band gap of the synthesized ZGON was estimated via the Tauc Plot ${ }^{[34]}$ method, after dispersing the ZGON nanoparticles in water. The band gap of the product with $R=5$ (urea/zinc) is calculated to be $2.3 \mathrm{eV}$, as in figure SI.11. Through the covalent bonding and dispersion of conduction and valence bands, the ZnO cooperated into GaN lattice reduces the band gap, which leads to a yellow color, as shown in figure SI.12.

\section{Dye decomposition tests}

To test the photo-catalytic property of ZGON, the synthesized powder nanoparticles were used in a model reaction to mimic the decomposition of organic pollutants (the organic degradation should give a period of stabilizing time to avoid the color reduction causing by chemical/physical absorption). For the purpose, the catalytic powder $(20 \mathrm{mg})$ was added to an aqueous solution of Rhodamine B (RhB, $20 \mathrm{ml}, 5 \mathrm{ppm}$ ) and let under stirring under UV light (250W). A significant color change was observed already after 1 hour, while after 2 hours no color was visible (see figure 7.A). For comparison, the decomposition of RhB was also tested in the presence of pure GaN but also in absence of any catalyst. As shown in figure 7.B, the concentration of RhB is decreasing with a faster rate when the bimetallic catalyst was added. 


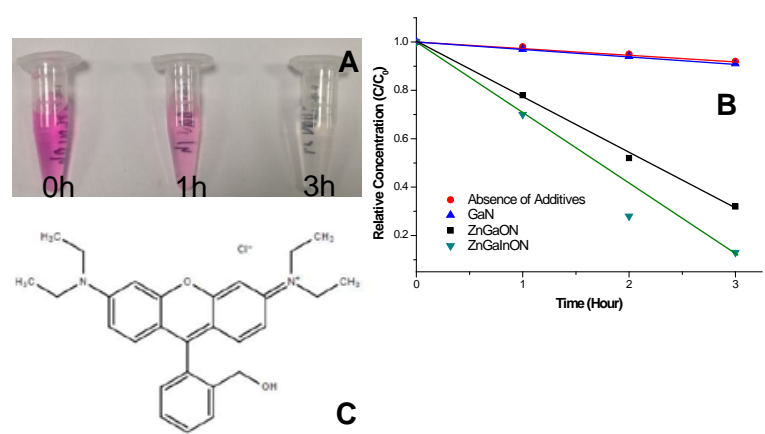

Figure 7. A) Concentration of Rhodamine B after UV exposure by measuring every hour the peak intensity in the RhB solution after UV exposure; B) Pictures of RhB solution samples after UV light exposure at different hours. C) Rhodamin B molecular structure. The straight lines are only guidelines for eyes.

In order to understand the absorption mechanism of RhB on the catalyst surface, the catalyst powder has been recovered after testing and an IR spectrum was recorded (figure SI.17). The spectra of pure RhB and catalyst before testing are also reported for comparison. The catalytic powder was thoroughly washed with water before recording the IR spectrum. From this figure peaks at $1217 \mathrm{~cm}^{-1}, 1364 \mathrm{~cm}^{-1}$ and $1738 \mathrm{~cm}^{-1}$ can be observed, corresponding to $\mathrm{CH}_{2}-\mathrm{OH}$ and $\mathrm{CH}_{2}-\mathrm{CH}_{3}$ and $\mathrm{C}=\mathrm{N}$ bonds, respectively. This indicates $\mathrm{RhB}$ is absorbed (physical absorption) electrostatically (possibly via van der Waals forces) onto the catalyst surface. Finally, the catalyst powder was washed with acetone and IR spectrum recorded again (figure S.I. 17) showing that all absorbed species were removed, thus ruling out any covalent bond formation and enabling us to reuse the powder for further testing. To prove that the removal of RhB takes place via catalytic decomposition and to rule out simple adsorbtion process, $\mathrm{RhB}$ was also tested in the dark, using the same synthetic condition. Results showed significant changes neither in the colour of the RhB solution (figure SI.18) nor in its structure, as ascertained by UV-vis (figure SI.18) and FT-IR (figure SI.19) spectra, recorded before and after the testing in the dark. Full RhB decomposition mechanism by the oxy-nitride is still under investigation. The stability of the catalysts was also proved by XRD study in the material before and after testing (figure SI.20, showing no significant differences.

\section{Water splitting}

Finally, the potential of the bi-metallic oxy-nitride was tested for photo-chemical water splitting and results reported in figure 8 . The evolution of hydrogen was $16 \mu \mathrm{mol} / \mathrm{h}$ with using $50 \mathrm{mg}$ photocatalysts, similar to what observed previously. ${ }^{[35]}$ But in the present case with room for improvements as pointed out by other authors, crystal imperfections strongly affect performances. ${ }^{[19]}$

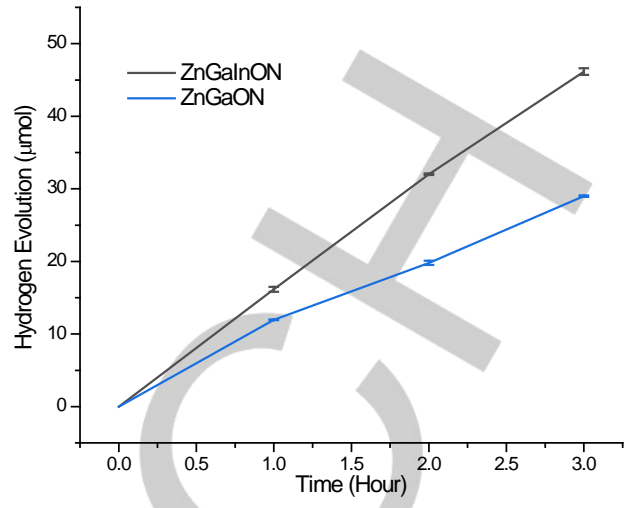

Figure 8. Hydrogen evolution by photocatalytic water splitting in the presence of ZGON and ZGION nanoparticles. Pt-based co-catalyst was added to the solution in form of 0.1 vol\% chloroplatinic acid (platinum source).

From the figure above, ZGON nanoparticle shows a catalytic efficiency with the hydrogen evolution rate of $12 \mu \mathrm{mol} / \mathrm{hour}$, while the rate is $16 \mu \mathrm{mol} / \mathrm{hour}$ for the ZGION catalyst. The reaction has been repeated for 5 times and the error bar is near $0.3 \%$, indicating both high photo-activity and reusability of the quaternary catalyst.

\section{Conclusions}

In summary, we have presented the synthesis of multi-metallic metal(s) oxy-nitride systems via a facile route. By using the urea glass route, we are able to control particle size and crystallinity. The urea amount in particular has a decisive effect. ZGON and ZGION nanoparticles with a band gap energy of $\sim 2.2 \mathrm{eV}$ and $\sim 1.9$ $\mathrm{eV}$, respectively (depending on the urea and metal ratio), were tested for the photo-catalytic decomposition of rhodamine B with good performance (reaction constant of $0.22 \mathrm{~h}^{-1}$ for ZCON and $0.29 \mathrm{~h}^{-1}$ for ZGION

\section{Experimental Section}

Gallium(III) nitrate hydrate (CAS 69365-72-6, crystalline, 99.9\% trace metals basis), zinc acetate (CAS 5970-45-6, 99.99\% trace metals basis), indium nitrate hydrate (CAS 207398-97-8, 99.99\% trace metals basis) and urea (CAS 57-13-6, ACS, Reag. Ph Eur) were purchased from SigmaAldrich, Inc. Ethanol (603-002-00-5, Absolute, 99.8\%) was purchased from Honeywell Inc.

In a standard synthesis, the metal precursors are dissolved in ethanol, then suitable amount of urea are added. The solution is stirred till homogeneity and let for $24 \mathrm{~h}$ to evaporate the excess of solvent. In this step a gel-like precursor is formed alongside a complex between urea and metals. ${ }^{[22]}$ The resulting "jelly" phase was transferred into a crucible and thermally treated up to $800^{\circ} \mathrm{C}$ for 5 hours, under nitrogen flow. After cooling down to room temperature, a yellow/orange powder is obtained. The process is exemplary shown in figure 9 for the preparation of ZGON. 

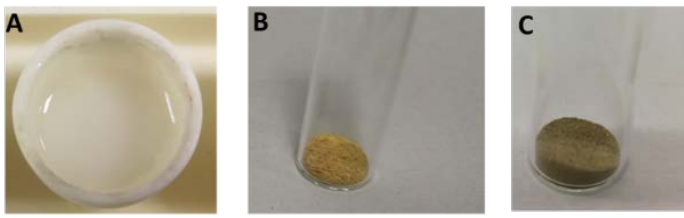

Figure 9. Pictures of $Z n G a: u r e a\left(R=10, R_{M}=2\right)$ sample before $(A)$ and after $(B)$ heat treatment. In $C$ a final ZGION sample $\left(R=10, R_{M}=2, R_{M}^{\prime}=0.2\right)$ is also shown.

To identify the best conditions but also to define the reaction mechanism, different metals ratio $\left(R_{M}\right)$ and urea/metal ratio $(R)$ has been explored. As discussed in the following, the best Ga:Zn molar ratio to achieve a crystalline and pure product was found to be $R_{M}=2$, while the best urea/metal ratio (urea:Zn) was found to be $R_{M}=10$. For the $Z G I O N$, the best In:Zn molar ratio was found to be $\mathrm{R}^{\prime} \mathrm{M}=0.2$. As shown in figure 9 . B-C, the colour of these products is the expected one. ${ }^{[4]}$ The lower reaction temperature compared to the one used by other routes, hinders the evaporation of $\mathrm{Zn}$, observed for treatment at $\mathrm{T}>950^{\circ} \mathrm{C} .{ }^{[36]}$ This point is especially important, because improved performances are expected for Zn-rich products. ${ }^{[4]}$

Specific details on the prepared nanoparticle systems are reported in table 1.

Table 1. Experimental details on the synthesized nanoparticles.

\begin{tabular}{|c|c|c|c|c|c|c|c|c|}
\hline Phase $^{\#}$ & $\begin{array}{c}R_{M} \\
(G a / Z n)\end{array}$ & $\begin{array}{c}R \\
\text { (Urea/Zn) }\end{array}$ & Surface area $\left(\mathrm{m}^{2} / \mathrm{g}\right)$ & Band gap (eV) & Size $(n m)^{*}$ & $\mathrm{D}(\mathrm{nm})^{\star *}$ & \multicolumn{2}{|c|}{ EA (w\%) } \\
\hline GaN & - & - & - & 3.2 & 14 & 8.2 & 12.99 & 0.46 \\
\hline ZnGaON & 2 & 15 & 54.3 & 2.1 & 13 & 6.2 & 6.79 & 0.16 \\
\hline ZnGaON & 1 & 15 & 34.5 & 2.3 & 14 & $\mathrm{~N} / \mathrm{M}$ & - & - \\
\hline ZnGaON & 1 & 10 & 36.7 & 2.4 & 19 & 12.5 & 8.40 & 0.30 \\
\hline ZnGalno.1ON & 1 & 10 & 28.9 & 2.1 & 15 & 29 & 9.78 & 0.18 \\
\hline $\mathrm{ZnGaln}_{0.2} \mathrm{ON}$ & 1 & 10 & 62.9 & 1.97 & 12 & $\mathrm{~N} / \mathrm{M}$ & 13.95 & 0.83 \\
\hline $\mathrm{ZnGaln}_{0.3 \mathrm{ON}}$ & 1 & 10 & 6.4 & 1.7 & 12.5 & 30.6 & 15.14 & 0.95 \\
\hline $\mathrm{ZnGaln}_{0.3 \mathrm{ON}}$ & 2 & 10 & 31.3 & 1.7 & 14 & 14.6 & 10.80 & 0.29 \\
\hline
\end{tabular}

For the photocatalytic tests, the photocatalytic decomposition of rhodamine $\mathrm{B}(\mathrm{RhB})$ was tested in the presence and in the absence of the photocatalyst. This reaction was chosen as model reaction to mimic the decomposition of dye pollutant. The catalytic powder $(50 \mathrm{mg})$ was added to an aqueous solution of $\mathrm{RhB}(5 \mu \mathrm{g} / \mathrm{L})$ and stirred vigorously. The dispersion was then placed in an incubator containing a $\mathrm{Hg}$-lamp (UV 250W Hand Lamp, $230 \mathrm{~V} / 50 \mathrm{~Hz}$, UV Light Technology Inc.) and let to be irradiated for 2 hours. An aliquot was taken every 20 minutes from the dispersion. The powder was then separated from the liquid part by centrifugation. The supernatant was analysed by UV-Vis spectroscopy to measure the corresponding spectrum of $\mathrm{RhB}$ in solution. A spectrum of the pure $\mathrm{RhB}$ solution in absence of catalysts and at the initial concentration was also recorded at the beginning for comparison.

\section{Acknowledgements}

SEM and TEM experimental data were provided by the Nanovision Department of the QMUL. XRD experimental data were provided by the Material Research Institute of the QMUL. $\mathrm{N}_{2}$ adsorption testing was provided by the STEBICEF Department,
Università di Palermo. C.G. and T.R. acknowledge the QMUL and Chinese Council Scholarship for financial support.

Keywords: Oxynitrides $\cdot$ dye decomposition $•$ urea glass route $•$ water splitting $\bullet$ multi metallic nitrides

[1] T. Takata, G. Hitoki, J. N. Kondo, M. Hara, H. Kobayashi, K. Domen, Visible-Light-Driven Photocatalytic Behavior of TantalumOxynitride and Nitride, 2007.

[2] F. Tessier, R. Marchand, J. Solid State Chem. 2003, 171, 143-151.

[3] M. Yashima, H. Yamada, K. Maeda, K. Domen, Chem. Commun. 2010, 46, 2379.

[4] K. Maeda, K. Teramura, T. Takata, M. Hara, N. Saito, K. Toda, Y. Inoue, H. Kobayashi, K. Domen, † Kazuhiko Maeda, et al., J. Phys. Chem. B 2005, 109, 20504-20510.

[5] M. Zhong, Y. Ma, P. Oleynikov, K. Domen, J.-J. J. Delaunay, Energy Environ. Sci. 2014, 7, 1693.

[6] W. Q. Han, Z. Liu, H. G. Yu, Appl. Phys. Lett. 2010, 96, 183112.

[7] P. Carrier, S. H. Wei, J. Appl. Phys. 2005, 97, 033707. 
[8] J. Wu, W. Walukiewicz, K. M. Yu, J. W. Ager, E. E. Haller, H. Lu, W. J. Schaff, Y. Saito, Y. Nanishi, Appl. Phys. Lett. 2002, 80, 39673969.

[9] K. Kamata, K. Maeda, D. Lu, Y. Kako, K. Domen, Chem. Phys. Lett. 2009, 470, 90-94.

[10] K. Lee, Y.-G. Lu, C.-H. Chuang, J. Ciston, G. Dukovic, J. Mater Chem. A 2016, 4, 2927-2935.

[11] Z. Wang, J. Han, Z. Li, M. Li, H. Wang, X. Zong, C. Li, Adv. Energy Mater. 2016, 6, 1-8.

[12] H. Chen, L. Wang, J. Bai, J. C. Hanson, J. B. Warren, J. T. Muckerman, E. Fujita, J. A. Rodriguez, J. Phys. Chem. C 2010, 114, 1809-1814.

[13] X. Sun, K. Maeda, M. Le Faucheur, K. Teramura, K. Domen, Appl Catal. A Gen. 2007, 327, 114-121.

[14] K. Lee, B. M. Tienes, M. B. Wilker, K. J. Schnitzenbaumer, G. Dukovic, Nano Lett. 2012, 12, 3268-3272.

[15] Á. Vass, Z. Pászti, S. Bálint, P. Németh, G. P. Szíjjártó, A. Tompos, E. Tálas, Mater. Res. Bull. 2016, 83, 65-76.

[16] J. Li, B. Liu, W. Yang, Y. Cho, X. Zhang, B. Dierre, T. Sekiguchi, A. Wu, X. Jiang, Nanoscale 2016, 8, 3694-3703.

[17] Y. L. Hu, Z. Wu, X. Zheng, N. Lin, Y. Yang, J. Zuo, D. Sun, C. Jiang, L. Sun, C. Lin, et al., J. Alloys Compd. 2017, 709, 42-53.

[18] Y. MASUBUCHI, J. Ceram. Soc. Japan 2013, 121, 142-149.

[19] B. Adeli, F. Taghipour, Appl. Catal. A Gen. 2016, 521, 250-258.

[20] D. P. Chen, S. E. Skrabalak, Inorg. Chem. 2016, 55, 3822-3828.

[21] A. Miyaake, Y. Masubuchi, T. Takeda, S. Kikkawa, Mater. Res. Bull. 2010, 45, 505-508.

[22] C. Giordano, C. Erpen, W. Yao, B. Milke, M. Antonietti, Chem. Mater. 2009, 21, 5136-5144.

[23] W. Lei, M. G. Willinger, M. Antonietti, C. Giordano, Chem. A Eur. J. 2015, 21, 18976-18982.

[24] F. Armetta, M. L. Saladino, C. Giordano, C. Defilippi, Ł. Marciniak, D. Hreniak, E. Caponetti, Sci. Rep. 2019, 9, 3368.

[25] K. Maeda, T. Takata, M. Hara, N. Saito, Y. Inoue, H. Kobayashi, K. Domen, J. Am. Chem. Soc. 2005, 127, 8286-8287.

[26] R. A. Young, Oxford Univ. Press 1993.

[27] L. Lutterotti, S. Gialanella, Acta Mater. 1998, 46, 101-110.

[28] M. A. Tagliente, L. Tapfer, P. Waltereit, O. Brandt, K. H. Ploog, J. Phys. D. Appl. Phys. 2003, 36, 192-197.

[29] F. M. Morales, D. González, J. G. Lozano, R. García, S. HauguthFrank, V. Lebedev, V. Cimalla, O. Ambacher, Acta Mater. 2009, 57, 5681-5692.

[30] F. Armetta, M. L. Saladino, D. F. Chillura Martino, P. Livreri, M. Berrettoni, E. Caponetti, J. Alloys Compd. 2017, 719, 264-270.

[31] E. Kirkendall, L. Thomassen, C. Uethegrove, Trans. Am. Inst. Min. Metall. Eng. 1939, 133, 186-203.

[32] C. Schliehe, C. Giordano, Nanoscale 2013, 5, 3235-9.

[33] C. Schliehe, J. Yuan, S. Glatzel, K. Siemensmeyer, K. Kiefer, C. Giordano, Chem. Mater. 2012, 24, 2716-2721.

[34] J. Tauc, Mater. Res. Bull. 1968, 3, 37-46.

[35] X. Wang, K. Maeda, A. Thomas, K. Takanabe, G. Xin, J. M. Carlsson, K. Domen, M. Antonietti, Nat. Mater. 2009, 8, 76-80.

[36] J. Q. Hu, X. L. Ma, Z. Y. Xie, N. B. Wong, C. S. Lee, S. T. Lee, Chem. Phys. Lett. 2001, 344, 97-100. 


\section{Entry for the Table of Contents}

TOC

\section{FULL PAPER}

$$
\underset{\substack{\text { Water } \\ \text { Spliting }}}{\longrightarrow}
$$

Schematic representation of the urea glass route.
T. Rao a, M.L. Saladino ${ }^{b}$, Y. Fang ${ }^{c}, X$. Wang ${ }^{c}$, C. Giordano*

Page No. - Page No.

Multimetallic Oxynitrides Nanoparticles for a New Generation of Photocatalysts 